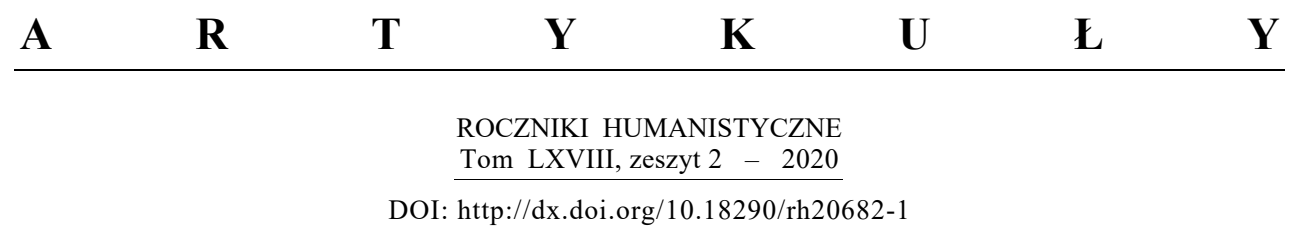

MARCIN KARKUT

\title{
DON JAIME MIGUEL DE GUZMÁN: EL MARQUÉS DE LA MINA Y SU CARRERA MILITAR
}

El marqués de la Mina fue uno de los representantes más destacados de la vida pública del siglo XVIII español. Como un militar ilustrado, político y embajador estuvo relacionado con el marqués de la Ensenada y su gran proyecto reformista ${ }^{1}$, como escritor militar dejó numerosos textos sobre las estrategias y tácticas o advertencias para los oficiales ${ }^{2}$. Según el profesor José Luis Gómez Urdáñez, a pesar de sus grandes virtudes y méritos, el marqués de la Mina sigue siendo un militar poco conocido, aún en la historiografía española ${ }^{3}$.

Dr. Marcin Karkut - Universidad Católica Juan Pablo II de Lublin, Instituto de Historia, Cátedra del Mundo Hispano, Política y Relaciones Internacionales, asistente; dirección de correspondencia — correo electrónico: marcin_karkut@hotmail.com; ORCID: https://orcid.org/00000002-2467-7361.

${ }^{1}$ J. L. Gómez URDAÑEZ, El proyecto reformista de Ensenada, Lleida: Milenio 1996.

${ }^{2}$ Ningún de estos textos fue publicado durante la vida del marqués, véase: MARQUÉS DE LA MinA, Máximas para la Guerra. Estudio introductorio y edición crítica de Manuel-Reyes García Hurtado, Madrid: Ministerio de Defensa 2006, p. 56.

${ }^{3}$ Existen tres biografías del marqués, véase A. CÁNOVAs DEL CASTILlo, Biografía del Marqués de la Mina, en: Memorias militares de D. Jaime Miguel de Guzmán Dávalos y Spinola, Marqués de la Mina, Duque de Palata, Conde de Pezuela de las Torres, Grande de España de primera clase, Caballero del Toisón y de Sancti Spiritus, San Genaro y Calatrava, Capitán General de los Ejércitos de S. M., Director General del Cuerpo de Dragones, etc., etc., etc., sobre la guerra de Cerdeña y Sicilia en los años de 1717 a 1720 y guerra de Lombardía en los de 1734 a 1736 publicadas a expensas del Teniente General Excmo. Sr. D. Eduardo Fernández San Román, Marqués de San Román, precedidas de una introducción y de la biografia del autor por el Excmo. Sr. D. Antonio Cánovas del Castillo y de un informe del Excmo. Sr. D. José Gómez de Arteche. Ilustradas con retratos, mapas, planos y estados demostrativos, Madrid: Real Academia de la Historia, 1898; F.-J. DE Vicente Algueró, El Marqués de la Mina, Capitán General de Cataluña (1742 y 1749-1767). El Gobierno del Principado a mediados del siglo XVIII, 1986. Es una tesis doctoral escrita bajo la su- 
El marqués de la Mina tuvo un gran conocimiento teórico del armamento, estrategias y tácticas militares, pero también dominó la práctica de los campos de batalla. Sin duda alguna se le puede poner en la misma línea con otros excelentes generales de la época como el duque de Montemar, el marqués de Lede o el conde de Gages, que fue admirado por el mismo Federico II el Grande, y esto es un aspecto muy interesante ${ }^{4}$. El marqués de la Mina participó en todas las campañas militares españolas de la primera mitad del siglo XVIII: en la Guerra de Sucesión Española (1701-1713), en la expedición de Sicilia y Cerdeña (1717-1720), en el asedio de Ceuta (1720), en un intento de reconquista de Gibraltar (1727), en la expedición a Oran (1732), en la Guerra de Sucesión Polaca (1733-1738) y en la Guerra de Sucesión Austriaca (1740-1748) ${ }^{5}$.

Jaime Miguel de Guzmán Dávalos Spínola Ramírez de Haro, más conocido como el segundo Marqués de la Mina, nació en Sevilla el día 15 de enero a las dos de la tarde del año 1690 y seis días más tarde fue bautizado por el arzobispo Jaime de Palafox y Cardona ${ }^{6}$. Desafortunadamente, no sabemos mucho sobre su vida infantil. Su padre, Pedro José de Guzmán Dávalos y Santillán ${ }^{7}$ (1650-1720), fue gobernador de Panamá con el rango del teniente general. En el 1681 el rey Carlos II le concedió el título del marqués de la Mina, gracias a sus méritos en el campo de batalla. La madre de Jaime Miguel, Juana María Spínola y Ramírez de Haro (1654-1698), provenía de una familia noble de la que su hijo heredó el título del Conde de Pezuela de las Torres ${ }^{8}$. Tenía el segundo Marqués de la Mina una hermana (a pesar de

pervisión del profesor Pedro Molas Ribalta y leída en la Universidad de Barcelona en el año 1986. Se ha publicado solo un resumen de la tesis: F.-J. de Vicente Algueró, El Marqués de la Mina, Capitán General de Cataluña (1749-1767), Barcelona, Universitat de Barcelona 1986; IDEM, "El Marqués de la Mina, de militar profesional a ilustrado periférico", en: Primer Congres d'historia Moderna de Catalunya, Barcelona: Universitat de Barcelona, vol. 2, 1984, pp. 89-100; E. BAREA Amorena, Jaime de Gúzman y Spínola, Capitán general de Cataluña, II marqués de la Mina, Alicante: Editorial Club Universitario, 2016.

${ }^{4}$ R. GARCía CARCÉl (dir.), Historia de España. Siglo XVIII. La España de los Borbones, Madrid: Cátedra 2002, p. 107.

${ }^{5}$ MARQuÉS de LA Mina, Máximas para la Guerra, p. 38.

${ }^{6}$ P.J. GuzMÁn Dávalos, Compendio Politico de documentos, advertencias, y maximas morales que hace el paternal amor del Marqués de la Mina a su hijo Dn. Jayme Miguel de Guzman, y Espinola, Ramirez de Haro, Sexto Conde de Pezuela, de las Torres, Cavallero del Orn de Calatrava, y Coronel de un Regim.to de Dragones, en el Eg.to de Estremadura, El Escorial, 1713, h. [1] r.

${ }^{7}$ Gracias a su padre Jaime Miguel perteneció a la familia noble de la que provino, entre otros, Juan Alonso Guzmán, el primer marqués de Niebla, el maestro y asesor del rey Enrique III (13791406), véase A. CÁNOVAS del CASTILlo, Biografia del Marqués de la Mina, vol. I, p. CCLXXXII.

${ }^{8}$ D. OZANAM, Les diplomates espagnols du XVIII siècle. Introduction et répertorire biografique (1700-1808), Madrid-Bordeaux: Casa de Velázquez 1998, p. 294. 
que Antonio Cánovas del Castillo, en la introducción a las Memorias Militares apuntó: "Por mi parte, no he tropezado con noticia alguna tocante a otros hermanos") ${ }^{9}$ que se llamaba Micaela María de Guzmán y Spínola y que se casó con Juan Gaspar de la Cueva y de Velasco Ramírez de Arellano, el XII duque de Alburquerque y el capitán de las Fuerzas Reales, con quien tuvo tres hijos ${ }^{10}$.

En el año 1695, cuando entonces el joven Jaime Miguel tenía apenas 5 años, su padre fue acusado y encarcelado en la fortaleza San Lorenzo Real de Chagres en Panamá. Aunque existen numerosas fuentes que tratan sobre la cuestión del proceso penal del primer Marqués de la Mina, el asunto sigue siendo impreciso ${ }^{11}$. Pedro José de Guzmán, gracias a su extraordinaria carrera militar, consiguió los cargos del gobernador de Panamá, presidente de la Audiencia Real y el capitán de las Fuerzas Reales en Panamá ${ }^{12}$. Por lo tanto sus poderes en la Tierra Firme fueron incuestionables. Un memorial anónimo nos cuenta que las acusaciones de sus enemigos tenían naturaleza económica, que el propio marqués lo explicaba como producto de la envidia de sus acusadores (entre ellos el obispo Diego Ladrón de Guevara, Fernando de la Riva, Jerónimo de Córdoba, Juana de Bolívar i Fernando de Echevers). En noviembre del 1693 el gobernador fue excomulgado, dos años más tarde, en agosto de $1695^{13}$, fue encerrado en Chagres. Su esposa, Juana María muere en el 1698 luchando contra los tribunales de justicia y dejando a sus hijos pequeños medio huérfanos con el padre acusado y encerrado. Finalmente, después de un proceso perdurable de siete meses (durante el que al gobernador se le pusieron más de 900 preguntas) el marqués fue liberado. En el año 1702 llega con sus hijos a la Península Ibérica ${ }^{14}$.

\footnotetext{
${ }^{9}$ A. CÁnovas del CAStillo, Biografia del Marqués de la Mina, pp. CCLXXXII- CCLXXXIII.

${ }^{10}$ F. FERnÁNDEZ DE BÉTHENCOURT, Historia genealógica y heráldica de la Monarquía Española, Casa Real y Grandes de España, Madrid: Enrique Teodoro 1920, pp. 352-353.

${ }^{11}$ Las fuentes más importantes que tratan sobre este asunto son: un manuscrito del año 1713 de Pedro José de Guzmán que ya habíamos citado antes, un documento del año 1700 que se halla en el archivo del marqués de Alcañices y que cita Antonio Cánovas del Castillo, véase A. CÁNOVAS DEL CAstillo, Biografia del Marqués de la Mina, p. CCLXXXIV. La fuente más extensa es un memorial anónimo del año 1709 y que reúne todos los discursos del proceso penal del marqués, véase: P. J. Guzmán Dávalos, Papeles varios del Marqués de la Mina, Biblioteca Capitular y Colombiana 1709, 58-1-32 fol. 1-54.

${ }^{12}$ MARQuÉS DE la Mina, Máximas para la Guerra, p. 27.

${ }^{13}$ Aunque Antonio Cánovas del Castillo apunta el 1694 como un año del arresto del marqués, véase A. CÁNOvas del CASTILlo, Biografía del Marqués de la Mina, p. CCLXXXIV.

${ }^{14}$ MARQUÉs de LA Mina, Máximas para la Guerra, pp. 29-31.
} 
Teniendo en cuenta todo lo que había sucedido, la educación ${ }^{15}$ del joven hijo del marqués fue extraordinaria. La ausencia del padre pudo haber hecho muchos daños a la personalidad y carácter de su hijo, así que el marqués escribió un libro lleno de advertencias y consejos. Esta gran obra de veinte capítulos fue pensada como un compendio de conocimientos y una guía para el joven Jaime Miguel. El autor se refiere a menudo al Viejo y Nuevo Testamento y a la gama completa de los pensadores latinos y griegos, dándose a conocer como un gran erudito. Habla mucho de la muerte segura que espera a todos los hombres, del camino de la salvación, de la ingratitud de los hombres (subrayando su inocencia y la injusticia que domina al mundo ${ }^{16}$ ), de la variabilidad de la fortuna, de la importancia de poseer amigos verdaderos, del amor, de la música y la poesía ${ }^{17}$.

En el año 1705, después de la etapa de educación precipitada, Jaime Miguel decidió continuar las tradiciones militares de su familia e ingresó en el ejército. Un año más tarde ya fue nombrado capitán del regimiento de caballería Órdanes ${ }^{18}$. En el 1707 participó en la batalla de Almansa, después en el sitio de Tortosa y de Campo Mayor en la frontera con Portugal (1712). A la temprana edad de 20 años fue nombrado coronel del Regimiento de los Dragones, que fue creado y financiado por él y que tomó nombre de Pezuela (y más tarde Lusitania) ${ }^{19}$.

Como ya hemos señalado, durante la Guerra de Sucesión Española, Jaime Miguel de Guzmán luchó al lado de las tropas borbónicas contra la coalición de los Habsburgo, ya que, en virtud del testamento del último rey de los Austrias, Carlos II, todo el imperio, donde no se ponía el sol, fue entregado a Felipe de Borbón, duque de Anjou. Durante este conflicto el papel del regimiento de los dragones del joven coronel Jaime Miguel de Guzmán fue, sin

\footnotetext{
${ }^{15}$ Tiene por maestro (por poco tiempo) al famoso historiador jesuita y uno de los fundadores de la Academia de la Lengua Española, Joseph Cassani, véase J. AlmiRAnTe, Bibliografia militar de España, Madrid: Imprenta y Fundición de Manuel Tello, 1876, p. 526.

${ }^{16}$ Dice el marqués: "No he cometido jamás en la voluntad acción que empañe mi honor, ni que manche la integridad en los empleos que he obtenido", véase P. J. GuZMÁn DÁvAlos, Papeles varios del Marqués de la Mina, h. [1] vto.

${ }^{17}$ MARQUÉs DE LA Mina, Máximas para la Guerra, p. 32.

${ }^{18}$ A. CÁnOvas del Castillo, Biografía del Marqués de la Mina, p. CCLXXXVI.

${ }^{19}$ D. OzAnAM, Les diplomates espagnols du XVIIII siècle, p. 294. En la hoja de servicio no vemos ninguna prueba, ¿por qué Jaime Miguel ascendió tan rápido? Lo explica un decreto real: "Por cuanto habiendo admitido la proposición hecha por el Conde de Pezuela de las Torres de levantar un regimiento de caballería sobre el pie de dragones, compuesto de tres escuadrones de a cuatro compañías cada uno, se le despachó título de coronel del tal regimiento en 21 de diciembre del año próximo pasado; vengo en mandar que se le guarden las honras y preminencias de su cargo", véase A. CÁnovas del Castillo, Biografia del Marqués de la Mina, pp. CCLXXXVI-CCLXXXVII.
} 
embargo, rudimentario ${ }^{20}$. A pesar de ello, los superiores ya se habían dado cuenta de que los dragones de Pezuela estaban comandados por un oficial excepcional. En la libreta de los servicios leemos: "Este coronel se halla en estado de proseguir su mérito. Es muy bueno para cualquier operación de guerra, con singular acierto y conducta, como lo ha acreditado en las muchas que se le han ofrecido, logrando el mayor aplauso por la distinción con que siempre ha obrado. Su aplicación y celo facilita mantener este regimiento en la mayor unión, gobierno y disciplina, siendo uno de los mejores entre los dragones. Y últimamente se encuentran en este oficial todas las circunstancias que hacen perfecto a un buen coronel, habiéndose enmendado con su capacidad clara de las travesuras que le causó la mocedad y poca experiencia en algún tiempo, de forma que al presente no tiene más objeto que el del real servicio, mereciendo por todos motivos la real atención de Su Majestad la exactitud con que procede" 21 .

Después de haber firmado un humillante tratado de Utrecht en el año 1713, el Reino de España trató de reconquistar los territorios perdidos durante la Guerra de Sucesión. Gracias a la segunda esposa del rey Felipe V, Isabel Farnesio, y su compatriota y jefe del gobierno, Giulio Alberoni, el reino organizó la expedición a Sicilia y Cerdeña (1717-1720). Aunque la campaña fue, desde el punto de vista militar, todo un éxito, España tuvo que devolver los territorios recién conquistados. La expedición se convirtió entonces, como dice John Lynch, en un gran gasto de dinero y de tiempo ${ }^{22}$.

En el año 1720 Jaime Miguel de Guzmán fue nombrado coronel brigadier, gracias a sus méritos durante la expedición a Sicilia y Cerdeña. Aquel tiempo conoció también a los hombres que pronto se convertirían en los ministros que iban a decidir en la política exterior e interior de España. Entre muchos, los más importantes fueron Pedro de Aranda, Ricardo Wall y sobre todo Zenón de Somodevilla, el Marqués de la Ensenada ${ }^{23}$. Los siguientes

\footnotetext{
${ }^{20}$ El regimiento participó en el asedio de Barcelona en el 1714, véase M. HERNÁNDEZ SÁNCHEZBarba, M. Alonso BacQuer (dir.), Historia social de las Fuerzas Armadas Españolas, Madrid: Alhambra 1986, p. 66.

${ }^{21}$ Regimiento de Dragones de Lusitania. Año de 1721. Libreta de los servicios, edad, nación y calidades de los oficiales vivos, reformados, sargentos y cadetes o soldados de distinción, que sirven en el Regimiento de Dragones de Lusitania, con la circunstancia de las prendas y defectos que les asisten, y se les han podido conocer hasta el día 31 de enero de 1721, y demás particularidades que se han experimentado, todo arreglado a diferentes informes, y a la práctica que se tiene de su obra, Archivo General de Simancas, Secretaría de Guerra, Legajo 2480, C ${ }^{\mathrm{o}}$ 1, fol. 1.

${ }^{22}$ J. LYNCH, La España del siglo XVIII, Barcelona: Crítica 1991, p. 74.

${ }^{23}$ C. GonzÁlez CaizÁn, C. TARACha, D. TÉllez Alarcia, Cartas desde Varsovia. Correspondencia privada del Conde de Aranda con Ricardo Wall (1760-1762), Lublin: Werset 2005, p. 105.
} 
años de la vida del II Marqués de la Mina (su padre, I Marqués de la Mina muere en el 1720) le entregan a Jaime Miguel de Guzmán más éxitos y ascensos. Ya como mariscal de campo (a partir del año 1728) participa en la expedición a Orán que se rinde en el año 1732 al Duque de Montemar ${ }^{24}$. Después de la rendición de Orán el Marqués de la Mina fue nombrado asistente y auxiliar del capitán general de los Ejércitos Reales de Aragón, y en el año 1733 , avanzó al rango de teniente general ${ }^{25}$.

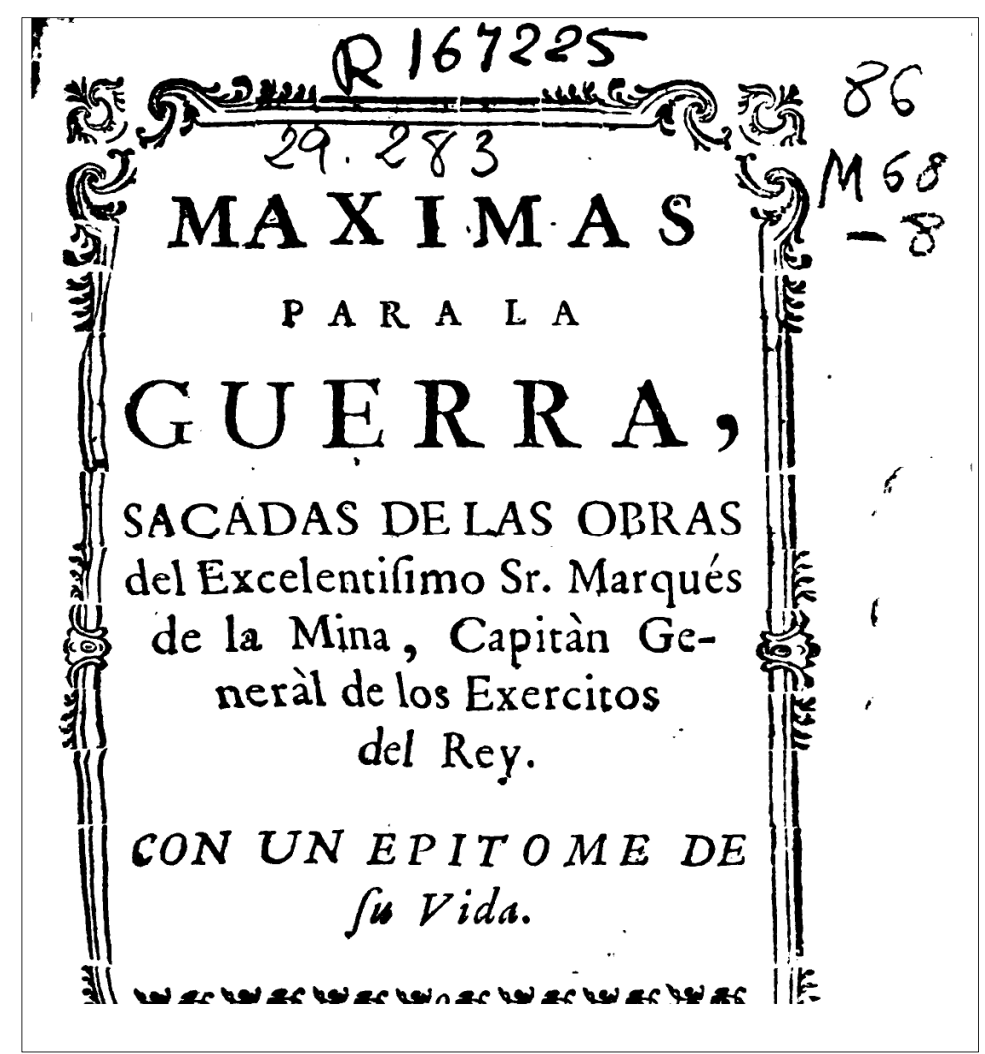

Ilustración 1. La portada de las Máximas para la Guerra, la obra maestra del Marqués de la Mina (https://books.google.es, 17.05.2016).

Otra guerra en la que participó el Reino de España en los años 17331738, conocida como la Guerra de la Sucesión Polaca, abrió la oportunidad para reconquistar las tierras italianas perdidas después de la Guerra de Suce-

\footnotetext{
${ }^{24}$ M. HeRnÁndez SÁnChEZ-BARBA, M. Alonso BACQUER (dir.), Historia Social de las Fuerzas Armadas Españolas, p. 84.

${ }^{25}$ D. OZAnAm, Les diplomates espagnols du XVIIII siècle, p. 294.
} 
sión Española. El Marqués de la Mina entonces opera ya como auxiliar del Duque de Montemar. Durante la victoriosa batalla de Bitonto en el año 1734 dirige al flanco derecho de los ejércitos reales. La derrota de los Austriacos terminó con un corto periodo de hegemonía en Italia y dejó a los españoles (y a Isabel Farnesio) poner en el trono de Sicilia y de Nápoles a su hijo mayor, Carlos (futuro Carlos III, rey de España) ${ }^{26}$.

En el año 1736 el Marqués de la Mina fue enviado a París para asumir la función de embajador plenipotenciario y extraordinario en la corte del rey Luis $\mathrm{XV}^{27}$. Durante su cargo firma en el nombre de su rey Felipe V el tratado de Viena (1738) y organiza el matrimonio entre Felipe, hijo menor del rey y de su segunda esposa Isabel Farnesio, con María Luisa, hija del rey Luis XV y su esposa polaca María Leszczyńska. Gracias a sus méritos en el campo diplomático ${ }^{28}$ el Marqués de la Mina obtiene la Insigne Orden del Toisón de Oro (1738) y la Orden del Espíritu Santo de que le otorga el rey Luis XV (1740). Este mismo año regresa a España donde, justo después de su llegada, es avanzado al rango del comandante general del regimiento de los dragones.

Cuando estalla la Guerra de Sucesión Austriaca, el Marqués de la Mina viaja a Italia (1742) reemplazando como comandante al Duque de Glimes ${ }^{29}$. Este mismo año conquista de forma espectacular el castillo de Aspermont ${ }^{30}$ y en el 1754 es nombrado capitán general ${ }^{31}$ del ejército español ${ }^{32}$.

El profesor Manuel-Reyes García Hurtado y otros historiadores dieciochistas, suelen constatar que gracias a la campaña del año 1744 y al plan de

${ }^{26}$ H. O’Donnell (dir.), C. Iglesias (coord.), Historia Militar de España. Edad Moderna, vol. III, Madrid: Ministerio de Defensa 2014, p. 138.

${ }^{27}$ J. BÉCKER Y GONZÁLEZ, "La Embajada del Marqués de la Mina (1736-1740)”, Boletín de la Real Academia de la Historia, vol. LXXXIII, Cuad. VI (diciembre 1923), pp. 364-378; vol. LXXXIV, Cuad. II (febrero 1924), p. 184-196; vol. LXXXIV, Cuad. IV (abril 1924), pp. 393-402; vol. LXXXV, Cuad. I (julio 1924), pp. 5-16; vol. LXXXVI, Cuad. I (enero - marzo 1925), pp. 42-115.

${ }^{28}$ Después de su estancia en París, trabajará dos veces más como embajador. En el año 1748 representa al Reino de España en Aquisgrán y en el año 1764 durante las negociaciones sobre la frontera hispano-francesa, véase: MARQUÉS DE LA MINA, Máximas para la Guerra, p. 40.

${ }^{29}$ Ibid., p. 38.

30 "Es el castillo de Aspermont un edificio cuadrado antiguo con una muralla de mucho espesor. Está colocado en un barranco de difícil acceso, aunque dominado por sus flancos de la pendiente de la montaña, sin terreno en donde situarse la artillería, de forma que pueda facilitar brecha practicable por su demasiada elevación, guardando sus costados dos precipicios que le sirven de profundos fosos, al abrigo de unos torreones en los ángulos del frente que los defiende de cualquier insulto, y por su entrada (que es más accesible) dos cortaduras con sus rastrillones que extendían sus fuegos a la imposibilidad del ataque que sólo por ese paraje podía ser regular". Ibid., p. 51.

${ }^{31}$ F. ANDÚJAR CASTILLO, "Capitanes generales y capitanías generales en el siglo XVIII", Revista de Historia Moderna: Anales de la Universidad de Alicante, vol. 22 (2004), pp. 7-78.

${ }^{32}$ D. OzAnAM, Les diplomates espagnols du XVIIII siècle, p. 294. 
la conquista de Piamonte, podemos, sin duda alguna, ponerle al Marqués de la Mina en la misma línea con los estrategas más excelentes de la época. Siempre decía el marqués y firmemente defendía su argumento que el sitio mejor para invadir el Piamonte (y luego Italia) es un espacio entre el Mediterráneo y los Alpes, a diferencia del Duque de Conti que intentaba demostrar la superioridad del argumento del ataque por Altos Alpes. Es imprescindible subrayar aquí que, cuando el Napoleón Bonaparte, al final del siglo, planeaba la invasión a Italia, utilizó las instrucciones del Marqués de la Mina ${ }^{33}$.

En el 1746 Mina es nombrado comandante general del ejército español de Italia $^{34}$, dos años más tarde, después del tratado de Aquisgrán $(1748)^{35}$ regresa a España y ocupa el cargo del capitán general del Ejercito Real de Cataluña. Se pone también al frente de la Audiencia Real de Cataluña ${ }^{36}$ hasta su muerte en el $1767^{37}$. Al unir el cargo del Capitán General y el Jefe de la Audiencia Real, que juntos constituían un tipo de diarquía ${ }^{38}$, el marqués obtuvo un poder inmenso. Sobre su cotidiano sus subordinados apuntaban:

Ocupa V. E. toda la mañana en despachos y audiencias, terminándola con la asistencia al cortejo y a la Misa....y le vean por las tardes en el activo reconocimiento de trabajos en Barceloneta, muelle, caminos, reparos de fortificación y demás obras públicas, autorizar seguidamente la comedia, ópera u otros espectáculos y, en fin, terminar las ocurrencias del día permitiéndose a la conversación y al juego hasta la hora del preciso retiro. ...dos o tres días a la semana a la diversión y el ejercicio de la caza ${ }^{39}$.

\footnotetext{
${ }^{33}$ MARQUÉS DE LA MiNA, Máximas para la Guerra, p. 40.

${ }^{34}$ C. DE FERNÁN NúÑEZ, La vida de Carlos III, Madrid: Fundación Universitaria Española 1988, pp. $69-70$.

${ }^{35}$ R. GARcía CARCÉl (dir.), Historia de España, pp. 132-133.

${ }^{36}$ M. DE los Ángeles PÉrez SAMPer, "La Audiencia de Cataluña en la edad moderna", Revista de Historia Moderna: Anales de la Universidad de Alicante, vol. 13/14 (1995), pp. 51-71; EADEM, "Los regentes de la Real Audiencia de Cataluña (1716-1808)", Pedralbes: Revista d'historia moderna, vol. 1 (1981), pp. 211-252; EADEM, "La Audiencia y el Capitán General de Cataluña", en: El mundo hispánico en el Siglo de las Luces, vol. II, Salamanca, Complutense 1994, pp. 1033-1050.

${ }^{37}$ D. Ozanam, Les diplomates espagnols du XVIIII siècle, p. 295.

${ }^{38}$ J.L. Comellas, Historia de España moderna y contemporánea (1474-1974), Madrid: Rialp 1974, p. 45.

${ }^{39}$ MARQuÉs de la Mina, Máximas para la Guerra, p. 41.
} 


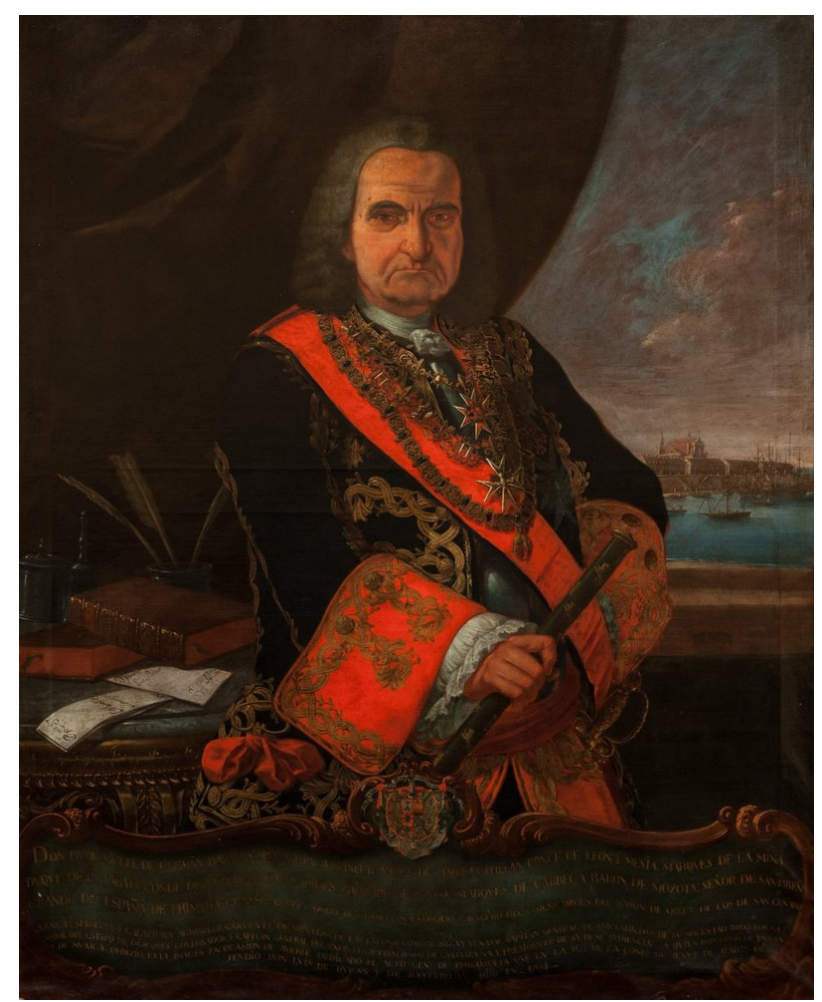

Ilustración 2. Manuel Tramulles Roig, Retrato del aristócrata español Jaime de Guzmán-Dávalos y Spínola (1690-1767), marqués de la Mina, conde de Pezuela de las Torres, teniente general del ejército español, capitán general de Cataluña y caballero de la Orden del Toisón de Oro, de la Orden del Espíritu Santo, de la Orden de San Miguel, de la Orden de Calatrava y de la Orden de Montesa, ca. 1760-1766, Óleo sobre lienzo, repr. Museu d'Història de Barcelona.

La importancia del marqués de la Mina creció aún más durante el reinado de Fernando VI (1746-1759). No se trata, sin embargo, de las actividades militares, sino de la política interna del Reino de España. Fernando VI, "el Pacífico" y su Secretario de Estado, José Carvajal y Lancaster fueron partidarios de la neutralidad española durante los conflictos europeos, ya que los militares tuvieron pocas oportunidades de demostrar sus habilidades. No obstante, el Marqués de la Mina sabía encontrarse en la nueva realidad política, en la que le ayudaron, por supuesto, las relaciones amistosas con Ensenada ${ }^{40}$.

Hemos señalado arriba que el Marqués de la Mina fue relacionado con la gran idea reformista del Marqués de la Ensenada. Fue un proyecto a grandí-

${ }^{40}$ J.L. GÓMEZ URDÁÑEZ, Fernando VI, Madrid: Arlanza 2001, p. 283. 
sima escala con objetivo de desarrollar económicamente el Estado que se encontraba en crisis a partir del reinado de los Austrias Menores ${ }^{41}$. En las reformas ensenadistas participaron los políticos relacionados con la corte de Fernando VI. Al frente del nuevo gobierno del rey estuvo, ya mencionado, José Carvajal y Lancaster (como el jefe de la Secretaría de Estado), Ensenada asumió la dirección de la Secretaría de Hacienda, Secretaría de Guerra y Secretaría de Marina e Indias. Un papel importante tuvo también el confesor real - padre Rávago (o, como dice el profesor Gómez Urdáñez - "más que un confesor") ${ }^{42}$. Sin embargo, un partidario más inapreciable de las reformas de Ensenada fue el Marqués de la Mina, su brazo derecho y gran amigo. Juntos trabajaron en organizar la Milicias Provinciales y en crear un catastro que fue un fundamento del sistema tributario más eficaz ${ }^{43}$.

Cuando en el año 1759 murió el rey Fernando VI y su hermanastro Carlos viajaba de Nápoles a España para ser coronado, el Marqués de la Mina se encontró al frente del cortejo de bienvenida al nuevo rey (se compuso el cortejo de los numerosos grandes españoles como: Duques de Medinaceli, Medinasidonia, Bournonville y Conde de Oñate). El Marqués de la Mina, el "soberano de Cataluña" ${ }^{44}$ proclamó entonces un solemne discurso:

Señor: Consigue Cataluña la envanecida dicha de haber preferido vuestra majestad sus orillas para la primera posesión de sus vastos Estados, que, dominando los mares con velocidad imponderable, le trae la Providencia, yo la honra de ofrecer los pies de vuestra majestad, con una de las mejores y más pobladas de sus provincias, la felicidad, el amor y los votos de cuantos vasallos la componen. Veneró vuestra majestad en sus menores años, infante don Carlos, mi respeto; le buscó duque de Parma mi feliz destino; le escoltó desde aquella ciudad mi cuidado, obedeciéndole subalterno como generalísimo de las gloriosas armas del rey, su padre (que está en el cielo), hasta Bitonto y Bari, dejándole coronado rey de Nápoles. Ahora, señor, por el más estimable influjo de mi estrella, soy el primer soldado español que recibe y se postra [ante] vuestra majestad como su rey. Dígnese vuestra majestad de admitir mis humildes tributos ${ }^{45}$.

\footnotetext{
${ }^{41}$ IDEM, El proyecto reformista de Ensenada.

${ }^{42}$ IDEM, Fernando VI, p. 52.

${ }^{43}$ M. Tuñón de Lara, J. Valdeón Baruque, A. Domínguez Ortiz, Historia Hiszpanii, przeł. Sz. Jędrusiak, Kraków, Universitas, 2007, p. 335.

44 "El rey de Cataluña", "el soberano de Cataluña", "el Señor de Cataluña", así le llamaron al marqués Pedro de Aranda y Ricardo Wall en su correspondencia privada, subrayando sus grandes poderes, véase C. GonzÁlez Caizán, C. TARACha, D. Téllez Alarcia, Cartas desde Varsovia, p. 104 y 120. También un famoso escritor y viajero inglés, Henry Swinburne admite: „He governed Catalonia many years, more like an independent sovreign, than like a subject invested with a delegated authority", véase H. SwINBURne, Travels through Spain in the years 1775 and 1776, London 1779 , p. 25.

${ }^{45}$ G. CARIDI, Carlos III. Un gran rey reformador en Nápoles y España, Madrid: La Esfera de los Libros 2015, pp. 256-257.
} 
Describiendo al Marqués de la Mina no podemos olvidarnos de su gran proyecto urbanístico de la ciudad de Barcelona y sus alrededores. Se construyó unas fortificaciones en Figueras, Gerona, Lérida, Tarragona y Hostalrich. Henry Swinburne describe en su obra todos los cambios urbanísticos de este tiempo ${ }^{46}$. Al gobernar Cataluña el Marqués de la Mina creó numerosas instituciones científicas y culturales, como el Colegio de Cirugía (1764) o Academia de Ciencias Naturales y Artes $(1765)^{47}$.

Jaime Miguel de Guzmán, II Marqués de la Mina murió el día 25 de enero del año 1767. A pesar de dos matrimonios no tuvo descendientes ${ }^{48}$. En su lápida sepulcral inscribieron un epitafio:

\author{
D.O.M. Hic Gusmannorum jacet Epitome \\ Exc. Dom. Marchio de la Mina, \\ Dux, Princeps, summus Imperator, Praeses, \\ In Acie Flumen, In Aula Flamen, \\ Obiit heu! Homo, at non abiit Heros \\ Cui Inscriptio, Virtus omnis \\ Die 30 Jan. \\ Anno 1768 R.I.P
}

\footnotetext{
${ }^{46}$ The port is handsome; the mole is all of hewn stone, a master-piece of solidity and convenience. Above is a platform for carriages; below, vast magazines, with a broad key reaching from the city gates to the lighthouse. This was done by the orders of the late Marquis de La Mina, captaingeneral of the principality, where his memory is held in greater veneration than at the court of Madrid [...] Great are the obligations Barcelona has to him, he cleaned and beautified its Streets, built useful edifices, and forwarded its trade and manufactures, without much extraordinary expence to the province; for he had more resources, and made money go farther than most other governors can do, or indeed with to do. On the neck of land that runs into the sea, and forms the port, he pulled down some fishermen's huts, and in 1752 began to build Barceloneta, a regular town, consisting of about two thousand brick houses, quarters for a regiment, and a church, in which his ashes are deposited under a monument [...], véase: H. SwINBURne, Travels through Spain, pp. 25-27; véase F.J. DE Vicente Algueró, „La «política educativa» del Marqués de la Mina, Capitán General de Catalunya (1754-1767)", en: Educación e Ilustración en España, Barcelona: niversitat de Barcelona 1984, p. 334.

${ }^{47}$ Marqués De La Mina, Máximas para la Guerra, p. 51.

${ }^{48}$ Se casó con Francisca Funes de Villapando (hija de José Pedro Funes de Villapando y Sanz de Latras, V conde de Atarés), después de su muerte se casó en el año 1733 con María Agustina Zapata, V Duquesa de la Plata y VIII Condesa Real, que murió en el año 1784. Le heredó su sobrino, Pedro de la Cueva y Velasco y Guzmán, véase. E. Mogrobejo, Diccionario hispanoamericano de heráldica, onomástica y genealogía, vol. LVII, Bilbao: Editorial Mogrobejo-Zabala 1995-2015, pp. 5-8.
} 


\section{BIBLIOGRAFIA}

Almirante José, Bibliografía militar de España, Madrid: Imprenta y Fundición de Manuel Tello 1876.

AndúJAR CASTILlo Francisco, "Capitanes generales y capitanías generales en el siglo XVIII", Revista de Historia Moderna: Anales de la Universidad de Alicante, vol. 22 (2004).

Barea Amorena E., Jaime de Gúzman y Spínola, Capitán general de Cataluña, II marqués de la Mina, Alicante: Editorial Club Universitario 2016.

BÉCKER y GonZÁLEZ Jerónimo, “La Embajada del marqués de la Mina (1736-1740)”, Boletín de la Real Academia de la Historia, vol. LXXXIII-LXXXVI, Madrid 1923-1925.

Cánovas del Castillo A., Biografía del Marqués de la Mina, en: Memorias militares de D. Jaime Miguel de Guzmán Dávalos y Spinola, Marqués de la Mina, Duque de Palata, Conde de Pezuela de las Torres, Grande de España de primera clase, Caballero del Toisón y de Sancti Spiritus, San Genaro y Calatrava, Capitán General de los Ejércitos de S. M., Director General del Cuerpo de Dragones, etc., etc., etc., sobre la guerra de Cerdeña y Sicilia en los años de 1717 a 1720 y guerra de Lombardía en los de 1734 a 1736 publicadas a expensas del Teniente General Excmo. Sr. D. Eduardo Fernández San Román, Marqués de San Román, precedidas de una introducción y de la biografía del autor por el Excmo. Sr. D. Antonio Cánovas del Castillo y de un informe del Excmo. Sr. D. José Gómez de Arteche. Ilustradas con retratos, mapas, planos y estados demostrativos, Madrid: Real Academia de la Historia 1898.

CARIDI Giuseppe, Carlos III. Un gran rey reformador en Nápoles y España, Madrid: La Esfera de los Libros 2015.

Comellas José Luis, Historia de España moderna y contemporánea (1474-1974), Madrid: Rialp 1974.

CONDE de FERnÁn NúÑEz, La vida de Carlos III, Madrid: Fundación Universitaria Española 1988

De los Ángeles Pérez Samper María, "Los regentes de la Real Audiencia de Cataluña (17161808)", Pedralbes: Revista d'historia moderna, vol. 1 (1981).

De los Ángeles Pérez SAMPer María, „La Audiencia y el Capitán General de Cataluña”, en: El mundo hispánico en el Siglo de las Luces, vol. II, Salamanca: Editorial Complutense 1994.

De los Ángeles Pérez SAmper María, "La Audiencia de Cataluña en la edad moderna", Revista de Historia Moderna: Anales de la Universidad de Alicante, vol. 13/14 (1995).

De Vicente Algueró Francisco José, "El Marqués de la Mina, de militar profesional a ilustrado periférico", en: Primer Congres d'Historia Moderna de Catalunya, vol. 2, Barcelona: Ediciones de la Universidad de Barcelona 1984, p. 89-100.

De Vicente Algueró Francisco José, "La «política educativa» del Marqués de la Mina, Capitán General de Catalunya (1754-1767)", en: Educación e Ilustración en España, Barcelona: Publicaciones del Departamento de Educación Comparada e Historia de la Educación. Universidad de Barcelona 1984.

De Vicente Algueró Francisco José., El Marqués de la Mina, Capitán General de Cataluña (1742 y 1749-1767). El Gobierno del Principado a mediados del siglo XVIII, tesis doctoral, Barcelona 1986.

De Vicente Algueró Francisco José, El Marqués de la Mina, Capitán General de Cataluña (1749-1767), Barcelona 1986.

FERnÁNDEZ DE BÉTHenCoURT Francisco, Historia genealógica y heráldica de la Monarquía Española, Casa Real y Grandes de España, Madrid: Enrique Teodoro 1920.

García CARCÉl Ricardo (dir.), Historia de España. Siglo XVIII. La España de los Borbones, Madrid: Cátedra 2002. 
GóMEZ URDAÑEz José Luis, El proyecto reformista de Ensenada, Lleida: Milenio 1996.

Gómez Urdañez José Luis, Fernando VI, Madrid: Arlanza 2001.

GonzÁlez Caizán Cristina, TARacha Cezary, Téllez Alarcia Diego, Cartas desde Varsovia. Correspondencia privada del Conde de Aranda con Ricardo Wall (1760-1762), Lublin: Werset 2005.

Guzmán DÁvalos Pedro José, Papeles varios del Marqués de la Mina, Biblioteca Capitular y Colombiana 1709.

GuZmán DÁvalos Pedro José, Compendio Político de documentos, advertencias, y maximas morales que hace el paternal amor del Marqués de la Mina a su hijo Dn. Jayme Miguel de Guzman, y Espinola, Ramirez de Haro, Sexto Conde de Pezuela, de las Torres, Cavallero del Orn de Calatrava, y Coronel de un Regim.to de Dragones, en el Eg.to de Estremadura, E1 Escorial 1713.

Hernández SÁnchez-Barba Mario, Alonso Bacquer Miguel (dir.), Historia Social de las Fuerzas Armadas Españolas, Madrid: Alhambra 1986.

LYNCH John, La España del siglo XVIII, Barcelona: Crítica 1991.

MARQuÉs de la MinA, Máximas para la Guerra. Estudio introductorio y edición crítica de Manuel-Reyes García Hurtado, Madrid: Ministerio de Defensa 2006.

Mogrobejo Endika, Diccionario hispanoamericano de heráldica, onomástica y genealogía, vol. LVII, Bilbao: Editorial Mogrobejo-Zabala 1995-2015.

O’Donnell Hugo J. (dir.), Iglesias Cano María (coord.), Historia Militar de España. Edad Moderna, vol. III, Madrid: Ministerio de Defensa 2014.

OZANAm Didier, Les diplomates espagnols du XVIII siècle. Introduction et répertorire biografique (1700-1808), Madrid-Bordeaux: Casa de Velázquez 1998.

Regimiento de Dragones de Lusitania. Año de 1721. Libreta de los servicios, edad, nación y calidades de los oficiales vivos, reformados, sargentos y cadetes o soldados de distinción, que sirven en el Regimiento de Dragones de Lusitania, con la circunstancia de las prendas y defectos que les asisten, y se les han podido conocer hasta el día 31 de enero de 1721, y demás particularidades que se han experimentado, todo arreglado a diferentes informes, y a la práctica que se tiene de su obra, Archivo General de Simancas.

SWINBURNE Henry, Travels through Spain in the years 1775 and 1776, London 1779.

TuÑón de Lara Manuel, Valdeón BaruQue J., Domínguez Ortiz A., Historia Hiszpanii, przeł. Sz. Jędrusiak, Kraków: Univeritas 2007.

\section{DON JAIME MIGUEL DE GUZMÁN: EL MARQUÉS DE LA MINA Y SU CARRERA MILITAR}

\section{Resumen}

El II marqués de la Mina fue uno de los militares más admirables del Siglo de las Luces que todavía está muy poco conocido en la historiografía europea. Su espléndida carrera militar empezó durante la Guerra de Sucesión Española (1701-1713), luego participó en todos los conflictos militares españoles de la primera mitad del siglo XVIII. En el año 1754 es nombrado capitán general de Cataluña y se pone en frente de la Audiencia Real. Muere sin descendencia en 1767 en Barcelona.

Palabras claves: Marqués de la Mina; España; siglo XVIII; historia militar. 


\section{DON JAIME MIGUEL DE GUZMÁN: MARKIZ DE LA MINA I JEGO KARIERA WOJSKOWA}

\section{Streszczenie}

II markiz de la Mina to jeden z najbardziej godnych podziwu wojskowych epoki Oświecenia, wciąż jednak mało znany w europejskiej historiografii. Jego wspaniała kariera wojskowa rozpoczęła się podczas wojny o sukcesję hiszpańską (1701-1713), następnie brał udział we wszystkich hiszpańskich konfliktach zbrojnych w pierwszej połowie XVIII wieku. W 1754 r. został mianowany kapitanem generalnym armii katalońskiej i zwierzchnikiem Audiencia Real, czyli sądu królewskiego. Zmarł bezpotomnie w 1767 r. w Barcelonie.

Słowa kluczowe: Markiz de la Mina; Hiszpania; XVIII wiek; historia wojskowości.

\section{DON JAIME MIGUEL DE GUZMÁN: MARQUIS OF LA MINA \\ AND HIS MILITARY CAREER}

\section{S u m mary}

The II marquis of la Mine was one of the Spanish officers most admirable in the Century of the Enlightenment who is still unknown in the European historiography. His most splendid military career started during the War of the Spanish Succession (1701-1713), later he participated in every Spanish military conflict in the first half of the $18^{\text {th }}$ century. In 1754 , he was appointed as a Captain General of a Spanish army and as a head of a Real Court of Catalonia. He died without descendents in 1767 in Barcelona.

Key words: Marquis of La Mina; Spain; $18^{\text {th }}$ century; military history. 